\title{
4.2. Dynamics of an Ectoparasite-host System
}

\section{Stefan M. JANION}

The adaptations of parasites can be classified into adaptations to their hosts and adaptation to the environment in which their hosts live. Hence, two types of habitats have been distinguished here: a primary habitat, which is the host itself, and a secondary habitat, in which the host occurs. There are many adaptations and interactions in the parasite-host system. Here we will discuss ecological adaptations in ectoparasites (fleas) related to the organization of host populations (rodents), thus the adaptation of parasites to the primary habitat.

Fleas spend varying amounts of time on their hosts, where they feed on their blood, or in the nests of the hosts, where their larval development occurs, except for some species, e.g. Tunga penetrans L. (Labunec et al., 1966a).

The number of fleas in nests and shelters of the host is very high, exceeding many times the number of hosts (Gershkovich, 1955). It has been found that the removal of all fleas from the fur of rodents at 24-hour intervals on five consecutive days of capture and release, had no noticeable effect on the proportion of rodents infested or on the number of fleas per host. The number of fleas on the hosts and the proportions of particular flea species remained unchanged throughout the removal experiment (Janion, 1962). It may be concluded, therefore, that during these five days rodents were in permanent contact with places of flea occurrence, and that the removal of fleas from the hosts did not reduce their numbers in host nests.

Essentially, host nests are an inexahaustible source of fleas. The number of fleas varies, however, depending on the number of hosts and their activity. High activity of hosts and their frequent leaving the nest account for a greater "attachment" of fleas to host bodies and a prolonged occurrence on them. For this reason, Ioff and Skalon (1954) and subsequently many other authors distinguished an ecotype of "fleas associated with the fur", that is, occurring longer on the host body, and an ecotype of "fleas associated with the nest", that is, spending more time in host nests than on the hosts themselves.

Host life style has an important effect of the infestation by fleas. For example, there are differences in infestation between migrant and resident rodents. The infestation of migrants is lower, and the species composition of fleas on them is different than on residents (Janion, $1960 \mathrm{a}, 1960 \mathrm{~b})$. 
It has also been shown that the mobility of hosts related to changes of nests, even within the same site, accounts for changes in the number of fleas on them. For example, moving from one nest to another during the first period of movements gives rise to a decline in the number of fleas in the fur. After a ten-day stay in the new nest, the number of fleas on the host reaches the level from the period preceding the change (Fig. 4.7) (Janion, 1968).

Factors determining the level of saturation of the habitat on the host, that is, affecting the number of fleas on the host, largely depend

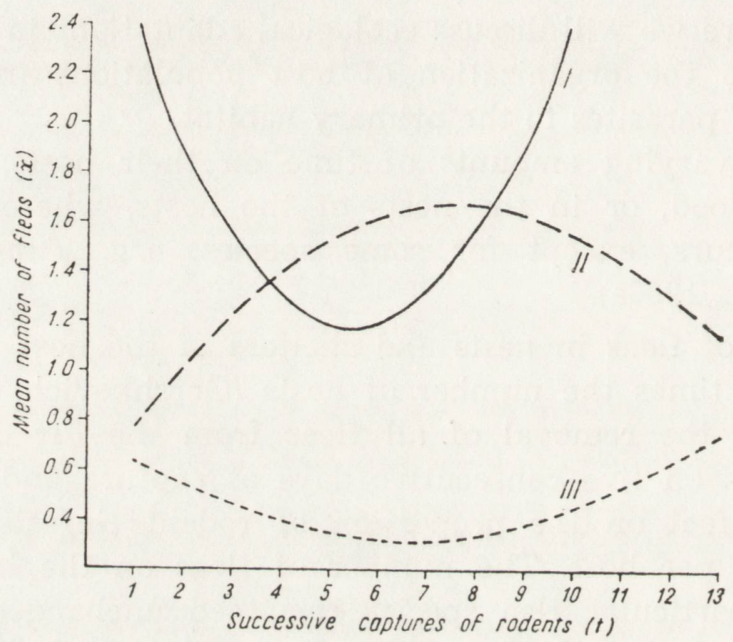

Fig. 4.7. Numbers of fleas on rodents at the continuous capture of fleas (curve II) and at their capture only in the first days of the experiment (curves I and III) (after Janion, 1968).

on the position of the host in the population structure, on the one hand, and on the number of fleas in the host environment, on the other (Janion, 1974). This can be illustrated by changes in the number and composition of fleas over the most lifetime in the wild (Figs. 4.8 and 4.9).

The age of a host, as determined by successive captures on the plot, can be divided into three periods with respect to the structure of infestation with fleas. The first period is when the young rodents leave the nest for the first time in search of food, thus begin to enter traps. At that time they are about 3-4 weeks old. The structure of infestation of these rodents with fleas differs from that in later periods. They are infested only with specific fleas, that is, fleas associated with only one host species. The second period is when the rodents are 4 to 10 weeks old. It is characterized by the appearance of fleas typical of 
foxes, squirrels, and birds, but not of rodents. In the third period, when rodents are 10 to 18 weeks old, nonspecific fleas do not occur on them, and a lower proportion of rodents is infested as compared with the two preceding periods. It is interesting that the rodents differing by age, that is, by the time of presence on the plot and from which fleas were collected on the same dates and hours of the day, showed age-specific differences in their infestation with fleas. This may imply that the individuals differring by age also differ by the places they visit on the plot, in which they are infested with fleas.

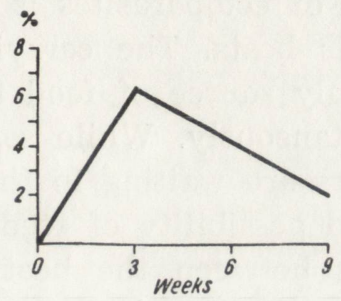

Fig. 4.8. The percentage proportion of nonspecific to specific fleas during August, September, October, Novernber, December, and January (after Janion, 1960b).

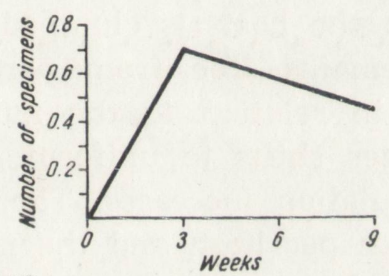

Fig. 4.9. Numbers of nonspecific fleas during August, September, October, November. December, and January (after Janion, 1960b).

The infestation of rodents is also sex-related. Usually males are more heavily infested than females (George \& Corbet, 1959; Smith, 1962; Brinck-Lindroth, 1968; Hass, 1969; Ulmanen \& Myllymäki; 1971, Peus, 1972; Mahnert, 1972). These authors suggest that the higher infestation of males is related to their higher activity during which they encounter more fleas, as compared with females. This is not a rule, however. Some data indicate that females can be more infested than males, and this depends on age, presence of the young in the nest, etc. (Janion, 1961).

There are also seasonal differences in the infestation of hosts. They are related to seasonal changes in the host population (Ioff \& Skalon, 1954; Rosicky, 1957; Skuratowicz, 1964). Other changes in infestation 
occur when human management causes seasonal disturbances in the habitat of rodents (Czerwińska, 1977).

Changes in the number of hosts, as already noted, are the equivalent of changes in the carrying capacity of flea habitat. An increase in the number of hosts means an increase in available food resources and in the number of nests, in which fleas can reproduce. The opposite is true when the number of hosts decreases. Remarkable changes, both annual and multiannual, in the number of rodents gave rise to the development of specific mechanisms of number regulation in their ectoparasites. As already noted, the number of ectoparasites is many times higher than the actual number of their hosts. The carrying capacity of the host population, which is the only source of food for parasites, is not used by all the parasites simultaneously. While some of them are feeding on the host body, the others are waiting in the habitat of the host for a contact with the host and possibility of feeding. There is a continual circulation of ectoparasites between the host habitat and host body. This process is influenced by the actual structure of the host population, and also by the relation between the size of the host population and flea population (Janion, 1979). In the case when the number of ectoparasites is not significantly higher than the number of their hosts, the distribution of parasites on the hosts is clumped. A certain proportion of the host population remains free from parasites. But when the number of hosts declines in relation to the number of ectoparasites, the distribution of the latter shifts to uniform. The proportion of infested animals in the population increases. The categories of infested rodents, number of parasites per host, and the number of not infested animals depend on the actual structure (organization) of the host population. When the number of hosts continue to decrease in relation to the number of fleas, the rate of the circulation of fleas between the host body and the environment is accelerated. This is the case because the carrying capacity of the host body is limited, and it can "feed" only a limited number of fleas at a time. Staying for a shorter time on the host, fleas suck less blood (Jurkina, 1968). This abbreviation of the host occupancy has serious consequences for female fleas. To reproduce, they need blood not only as food but also as the source of hormones secreted by the host adrenal glands. To get an adequate amount of these hormones, they must spend a sufficiently long time on the host. Otherwise the development of their eggs is inhibited or the eggs undergo resorption (Rotshild \& Ford, 1966). Thus, the females that are short of hormones do not lay eggs. The reproduction is inhibited and the size of flea populations is reduced. 
This summary of some relationships between rodents and fleas shows that ecological adaptations which evolved in the parasite-host system are highly diversified.

\subsection{Ecological Characteristics of Parasitic Worm (Helminth) Communities}

\section{Krystyna KISIELEWSKA}

\subsubsection{General Characteristics of Helminths in Bank Voles}

To date, about 80 species of helminths associated with the bank vole have been recorded in the literature over its geographical range. Recent, comprehensive papers concerning taxonomic revision of helminths (mostly nematods) in rodents introduced significant taxonomic changes. A review of these is beyond the scope of this paper and the numerical data given below on the helminth fauna of the bank vole are therefore approximate:

1) 33 species of Nematoda (16 genera, 7 families) - $42 \%$

2) 21 species of Cestoda (7 genera, 3 families) $-26 \%$

3) 16 species of Cestoda-larvae (6 genera, 1 family) $-20 \%$

4) 9 species of Trematoda (7 genera, 6 families) $-11 \%$

5) 1 species of Acanthocephala - 1\%

Thus, the helminth fauna of the bank vole is dominated by nematodes, and their dominance would be even higher $(52 \%$ of the community) if larval forms of nematodes were eliminated from the calculations, though they are only a taxonomic and not an ecological component of the helminth community.

Most frequently noted in bank voles of at least four European. countries are:

Trematoda: Plagiorchis muris (Tanabo 1922)

Cestoda: Catenotaenia pusilla (Goeze, 1782)

Catenotaenia cricetorum (Kirschenblatt, 1949)

Skrjabinotaenia lobata (Bear, 1925)

Aprostatandrya macrocephala (Douthidt, 1915)

Andrya microti (Erhardova, 1956)

Paranoplecephala brevis (Kirschenblatt, 1938)

Hymenolepsis diminuta (Rudolphi, 1819) 